OPEN ACCESS

Edited by: Vinicius Cotta-de-Almeida, Oswaldo Cruz Foundation, Brazil

Reviewed by:

Janis K. Burkhardt, Children's Hospital of Philadelphia,

USA

Shulamit Katzav

Hebrew University of Jerusalem, Israel

*Correspondence:

Noah Isakov,

The Shraga Segal Department of

Microbiology, Immunology and

Genetics, Faculty of Health Sciences,

Ben Gurion University of the Negev,

P.O.B. 653, Beer Sheva 84105, Israel noah@bgu.ac.il

Specialty section:

This article was submitted to T Cell Biology, a section of the journal

Frontiers in Immunology

Received: 30 July 2015 Accepted: 18 September 2015

Published: 05 October 2015

Citation:

Braiman A and lsakov N (2015) The role of Crk adaptor proteins in T-cell adhesion and migration. Front. Immunol. 6:509.

doi: 10.3389/fimmu.2015.00509

\section{The role of Crk adaptor proteins in T-cell adhesion and migration}

\author{
Alex Braiman ${ }^{1}$ and Noah Isakov ${ }^{1,2 *}$ \\ ${ }^{1}$ The Shraga Segal Department of Microbiology, Immunology and Genetics, Faculty of Health Sciences, The Cancer \\ Research Center, Ben Gurion University of the Negev, Beer Sheva, Israel, ${ }^{2}$ School of Pharmacy, University of Otago, \\ Dunedin, New Zealand
}

Crk adaptor proteins are key players in signal transduction from a variety of cell surface receptors. They are involved in early steps of lymphocyte activation through their $\mathrm{SH} 2-$ mediated transient interaction with signal transducing effector molecules, such as Cbl, ZAP-70, CasL, and STAT5. In addition, they constitutively associate, via their SH3 domain, with effector molecules, such as C3G, that mediate cell adhesion and regulate lymphocyte extravasation and recruitment to sites of inflammation. Recent studies demonstrated that the conformation and function of Crkll is subjected to a regulation by immunophilins, which also affect Crkll-dependent T-cell adhesion to fibronectin and migration toward chemokines. This article addresses mechanisms that regulate Crkll conformation and function, in general, and emphasizes the role of Crk proteins in receptor-coupled signaling pathways that control T-lymphocyte adhesion and migration to inflammatory sites.

Keywords: Crk adaptor proteins, signal transduction, $T$ lymphocytes, cell adhesion, cell migration, immunophilins, cyclophilin A, cyclosporin A

\section{Introduction}

Adaptor proteins are essential components of signal transduction pathways in all cell types due to their ability to link engaged receptors to specific downstream signaling cascades. The presence of multiple protein-protein interaction domains in an adaptor protein enables the simultaneous interaction with two or more effector molecules and the spatially and temporally orchestrated assembly of multimolecular complexes that are essential for signal transduction.

The mammalian CT10 (chicken tumor virus number 10) regulator of kinase (Crk) adaptor proteins, CrkI and CrkII, are alternatively spliced products of a single gene (1). Both proteins include an N-terminal Src homology 2 (SH2) domain followed by a C-terminal SH3 domain (2-4). CrkII includes an additional SH3 domain at the C-terminus ( $\mathrm{SH} 3 \mathrm{C}$ ), which is separated from the $\mathrm{SH} 3 \mathrm{~N}$ by a 47-residue-containing spacer region. A third member of the family, termed Crk-like (CrkL), is encoded by a closely related gene and possesses an overall structure similar to that of CrkII (2-4).

The Crk proteins are ubiquitously expressed and act as major convergence points of tyrosine kinase signaling pathways. They utilize their $\mathrm{SH} 2$ and $\mathrm{SH} 3$ domains for interaction with multiple effector molecules and integrate molecular information obtained from diverse sources, such as growth factors, extracellular matrix (ECM), pathogens, and apoptotic cells (2-6). In general, Crk proteins play an essential role in the regulation of immune cell functions leading to cell adhesion and migration, proliferation, differentiation, and apoptosis (2-6). 


\section{The Structure and Binding Partners of Crk}

Crk proteins possess two types of basic modules that function as protein-protein interaction domains. The Crk amino-terminus SH2 domain binds tyrosine-phosphorylated proteins containing the preferred consensus motif, pY-x-x-P, whereby specificity is provided by the two to five residues $\mathrm{C}$-terminal of the phosphotyrosyl group (7) (Figure 1). In accordance, Crk proteins interact via their $\mathrm{SH} 2$ domain with a large number of T-lymphocyte effector proteins, including the Crk-associated substrate lymphocyte type [p105CasL, also known as human enhancer of filamentation protein 1 (HEF1) or NEDD9] (8), Casitas B-lineage lymphoma (c$\mathrm{Cbl})(9,10)$, and $\zeta$-chain-associated protein kinase $70 \mathrm{kDa}$ (ZAP70) $(11,12)$ (Figure 2).

A second module found in all Crk proteins is an $\mathrm{SH} 3$ domain, which binds polyproline-rich sequences, with a preference toward

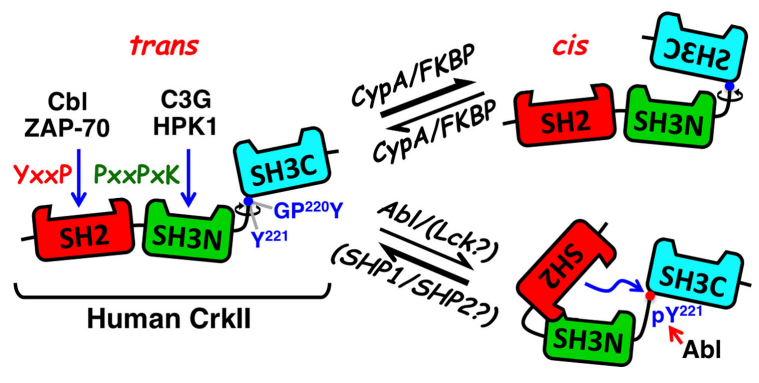

FIGURE 1 | Schematic structure and mode of regulation of CrkIl. The Crkll protein contains two protein-protein interaction domains and a single regulatory domain. The $\mathrm{N}$-terminal $\mathrm{Crkll-SH} 2$ domain (red) transiently interacts with phosphorylated tyrosine-containing peptide sequences possessing a pY-X-X-P consensus motif, which is found in signaling proteins, such as tyrosine-phosphorylated $\mathrm{Cbl}$ and ZAP-70. The Crkll-SH3N domain (green) interacts with polyproline-rich sequences in proteins such as C3G and HPK1, with a preference toward the P-x-L-P-x-K motif. The Crkll-SH3C (light blue) is an atypical $\mathrm{SH} 3$ domain due to the presence of hydrophobic, instead of aromatic, residues in its polyproline binding pocket, which reduces its affinity to polyproline-containing peptides. It functions predominantly as a regulatory region, which under certain conditions can block ligand interaction with Crkll. A 47-residue-containing spacer region, which links the two $\mathrm{SH} 3$ domains, contains a tyrosine residue (Tyr 221) that is a target for phosphorylation by $\mathrm{Abl}$, in ligand-stimulated cells. Phospho-Tyr221 can intramolecularly interact with, and block the $\mathrm{SH} 2$ domain, and induce a conformational change that alters Crkll accessibility to other potential ligands. A second motif in the linker region is a substrate for the peptidyl-prolyl cis-trans isomerases (PPlases), such as cyclophilin A and FK506, which can catalyze the cis-trans isomerization of the Gly-Pro ${ }^{220}$ peptide bond and interconverts Crkll between two distinct conformations. The Crkll cis isomer adopts a closed, autoinhibited conformation, while the Crkll trans isomer adopts an open, uninhibited conformation, which is available for interaction with $\mathrm{SH} 2$ and $\mathrm{SH} 3$ binding partners. Blue arrows indicate sites of interaction and a red arrow indicates a site of phosphorylation. Abl, Abelson murine leukemia viral oncogene; C3G, Cbl, Casitas B-lineage lymphoma; CRK, SH3 domain-binding guanine nucleotide-releasing factor; Crk, CT10 (chicken tumor virus number 10) regulator of kinase; CypA, cyclophilin A; FKBP, FK506 binding protein; GPY, Gly-Pro-Tyr motif; HPK1, hematopoietic progenitor kinase 1; Lck, lymphocyte-specific protein tyrosine kinase; pY, phosphotyrosine; SH2, Src homology 2; SH3C, C-terminal Src homology domain 3; SH3N, N-terminal Src homology domain 3; PPlase, peptidyl-prolyl cis-trans isomerase; SHP1, SH2 domain-containing protein tyrosine phosphatase 1; SHP2, SH2 domain-containing protein tyrosine phosphatase 2; Y, tyrosine residue; ZAP-70, zeta-chain-associated protein kinase 70. the P-x-L-P-x-K motif (17) (Figure 1). In T cells, this domain interacts with proteins such as the Crk SH3N domain-binding guanine nucleotide-releasing factor $(\mathrm{C} 3 \mathrm{G})(9,18)$ and the hematopoietic progenitor kinase 1 (HPK1) $(19,20)$ (Figure 2). It has been suggested that the CrkI/II- and CrkL-SH3N domains exhibit redundant substrate specificity and constitutively interact with their ligands, independent of the cell activation state $(21,22)$. Both SH2 and SH3 domains of CrkII interact with multiple binding partners in different cell types (2-6), although information about such interactions in $\mathrm{T}$ cells is relatively limited.

The SH3C of both CrkII and CrkL possesses a consensus $\mathrm{SH} 3$ sequence homology, but its binding surface contains some hydrophobic residues that substantially reduce its affinity to polyproline-rich sequences (23). Two independent studies have indicated that the Crk-SH3C domain contains a nuclear export signal that interacts with the Crm1/Exportin $(24,25)$. However, the physiological relevance of this low-affinity interaction has yet to be determined.

More recent studies suggested that the main function of the SH3C domain is directed at stabilizing CrkII and/or CrkL in a conformation that negatively regulates their biological function (25-29).

\section{Conformation, Function, and Expression Regulation of Crk Adaptor Proteins}

Apart from the $\mathrm{SH} 2$ and $\mathrm{SH} 3$ domains, Crk proteins contain additional regulatory motifs that may have an impact on the protein conformation and biological activity (Figure 1). One such motif includes a tyrosine residue within the linker region tethering the two SH3 domains (Tyr221 and Tyr207 in the human CrkII and CrkL, respectively) $(30,31)$. This tyrosine undergoes phosphorylation by a protein tyrosine kinase (PTK), such as Abl, following the interaction of a number of different ligands with their cognate cell surface receptors $(31,32)$. In addition, Tyr207 in CrkL is intensely phosphorylated by the oncogenic tyrosine kinase Bcr-Abl in Philadelphia chromosome-positive leukemia $(30,32)$, where CrkL is required for the Bcr-Abl-induced cell transformation (33).

The conserved tyrosine residues in CrkII and CrkL reside within a Y-x-X-P sequence, and upon phosphorylation, they internally interact with the $\mathrm{SH} 2$ domain, thus operating as dominantnegative binding motifs (32). NMR spectrometry analysis confirmed that phospho-Tyr221 in CrkII does indeed interact with the adjacent CrkII-SH2 domain and alters the protein's structure (34). The ensuing conformational change is expected to block the $\mathrm{SH} 3 \mathrm{~N}$, in addition to the $\mathrm{SH} 2$ domain, releasing CrkII from its binding partners and repressing CrkII-mediated signaling (32, 34).

A second regulatory motif within an extended loop in the $\mathrm{SH} 2$ domain possesses a proline-rich region, which is dispensable for phosphopeptide binding and is able to interact with various $\mathrm{SH} 3$ domains, including that of the Abl PTK $(32,35,36)$. Although the in vitro interaction of the full-length non-phosphorylated CrkII with Abl SH3 domain was rather weak, phosphorylation of CrkII-Tyr221, or addition of a phosphopeptide corresponding to the CrkII-Tyr221 phosphorylation site, stimulated CrkII 


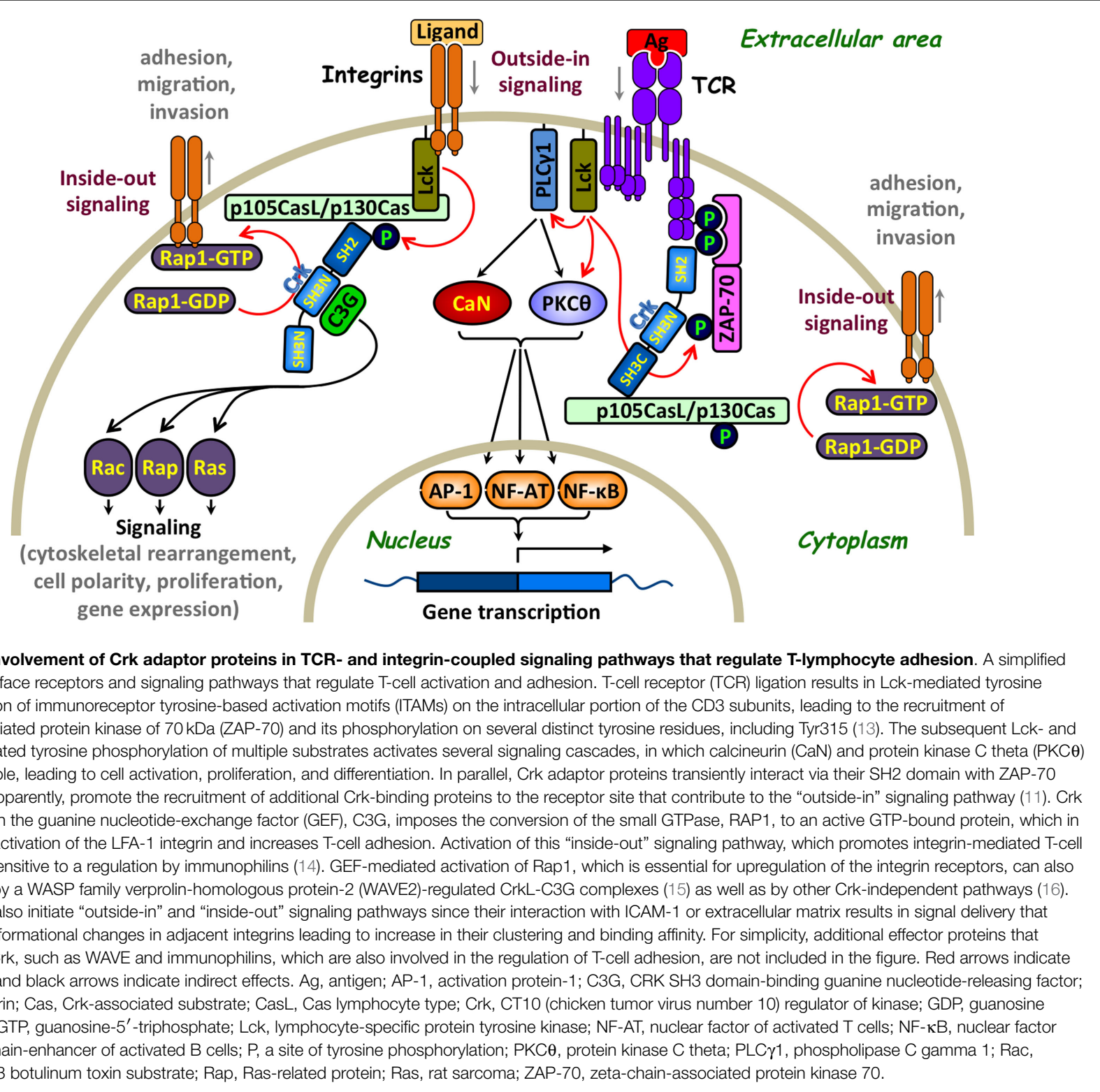

association with the Abl SH3 domain. The results suggested that the proline-rich insert within the Crk SH2 domain constitutes an $\mathrm{SH} 3$ domain-binding motif which is regulated by phosphopeptide ligand binding to the $\mathrm{Crk} \mathrm{SH} 2$ domain. CrkI possesses an identical SH3N domain allowing it to associate with Abl, but due to the lack of the linker region that includes Tyr221 (in addition to the entire SH3C domain), it cannot undergo phosphorylation in vivo, and apparently exhibits a very low affinity to the $\mathrm{Abl} \mathrm{SH} 3$ domain.

Recent studies revealed an additional regulatory motif within the linker region of the chicken, but not human, CrkII, which serves as a target for peptidyl-prolyl cis-trans isomerases (PPIases) $(28,29,37,38)$. Interconversion between the cis and trans isomers of the chicken CrkII occurred at the Gly237-Pro238 prolyl bond and was catalyzed by the PPIase, cyclophilin A (CypA). Acquisition of a cis conformation resulted in an intramolecular interaction between the two SH3 domains of CrkII, forming a closed conformation of an autoinhibited molecule. In contrast, the CrkII trans conformer acquired a relatively open and extended conformation that was readily available for interaction with $\mathrm{SH} 2$ and SH3N-binding partners. PPIases catalyze both cis-to-trans and trans-to-cis interconversion of CrkII, thereby determining the temporal and spatial activation of CrkII and CrkII-regulated signaling events.

More recent studies demonstrated that human T-cell-derived CrkII, but not CrkI, can physically interact with representatives of two families of PPIases, CypA and FK506 binding protein (FKBP), collectively termed immunophilins (14). In vitro coincubation of CypA with human T-cell-derived CrkII increased CrkII binding to $\mathrm{C} 3 \mathrm{G}$ while immunophilin inhibitors [such as cyclosporine A (CsA) and FK506] downregulated CrkII binding to $\mathrm{C} 3 \mathrm{G}$, suggesting that $\mathrm{CrkII-C} 3 \mathrm{G}$ interaction may be subjected 
to a regulation by immunophilins (14). This assumption was supported by fluorescence resonance energy transfer (FRET) studies of Jurkat $\mathrm{T}$ cells transfected with PICCHUx, a chimeric plasmid encoding the human $\mathrm{CrkII}_{1-236}$ sandwiched between cyan fluorescent protein (CFP) and yellow fluorescent protein (YFP). The basal FRET level of these cells was further augmented by CsA and FK506 treatment, reflecting a drug-induced inhibition of immunophilins and a trans-to-cis conversion of CrkII.

The differential sensitivity of the truncated chicken vs. human CrkII to PPIases was attributed to the nature of the amino acid that immediately follows the Pro238 (chicken)/237 (human) residue, Phe and Ile in the chicken and the human CrkII, respectively $(28,29,38)$. Since PICCHUx encodes a truncated human CrkII, which lacks Pro237, we predict that the Gly219-Pro220 motif plus its adjacent Tyr221 residue (an aromatic amino acid that is structurally related to Phe, except for a singly extra hydroxyl group) may be the site of immunophilin-mediated isomerization of the human CrkII.

CrkII shares with CrkL a high degree of sequence homology and ligand-binding preference, but the two proteins have distinct physiological roles. Structural studies demonstrated that the two proteins exhibit a distinct intramolecular assembly of their domains $(39,40)$, which might explain the differences in their biological activities. Furthermore, CrkL, unlike CrkII, lacks an immunophilin substrate motif.

Another line of studies demonstrated that the extent of expression of Crk is subjected to a regulation by microRNA (miRNA) (41). These studies were based on bioinformatic analyses predicting that Crk can be directly targeted by miR-126. Indeed, analysis performed in HSC-T6 cells (an immortalized rat liver stellate cell line) revealed that the Crk $3^{\prime}$ UTR is a direct target for miR-126. Furthermore, miR-126 overexpression led to decreased Crk protein expression, suggesting that Crk function and Crkdependent signaling pathways may also be regulated at the Crk protein expression level.

\section{Crk-Mediated Regulation of T-Cell Adhesion and Motility}

The ability of T lymphocytes to physically interact with antigenpresenting cells (APCs) and adhere to vascular endothelial cells is absolutely essential for the induction of all types of T-cellmediated immune responses. Accordingly, deficiency in adhesion molecules and/or their signaling pathways, such as in leukocyte adhesion deficiency (LAD), results in impaired or complete absence of immune responses (42). Interaction of T cells with APCs is a prerequisite for antigen recognition by the Tcell antigen receptor (TCR) and the subsequent activation and differentiation of the $\mathrm{T}$ cells into functional effector cells (43). In addition, migration of $\mathrm{T}$ cells to sites of inflammation is dependent on their ability to adhere to endothelial cells as the first step of extravasation and subsequent migration (44). Adhesion of $\mathrm{T}$ cells is mediated by members of the integrin family of receptors, such as lymphocyte function-associated antigen 1 (LFA-1; CD11a/CD18) and very late antigen-4 (VLA-4, $\alpha 4 \beta 1$ integrin), and additional receptors, such as L-selectins, CD44, and CD31 (44).
A large number of T-cell effector molecules play a role in the transduction of signals regulating the activity of adhesion receptors $(15,45-47)$. C3G is the first protein identified as being associated with Crk in a constitutive manner and is involved in cell adhesion $(48,49)$. It catalyzes guanosine- 5 -triphosphate (GTP) exchange by the small G-protein that removes GDP from small GTPases, such as Rap-1, Rap-2, and R-Ras, allowing their spontaneous reloading with GTP (50). These findings were initially observed in fibroblasts or epithelial cells and later on confirmed in other cell types, including lymphocytes. The targets of Rap-1 have been implicated in cell proliferation, cytoskeletal reorganization, and cell-to-cell contact (50-52). One of the most important biological effects of the Crk-C3G-Rap1 signaling pathway is the regulation of integrins, which mediate adherence of hematopoietic cells to ECM and stromal cells. The recruitment of C3G to Crk and subsequent loading of Rap1 with GTP increases the affinity of $\beta 1$-integrins to the $\operatorname{ECM}(51,53)$. This CrkL-C3G complex is required for a TCR-coupled signaling pathway, which involves the WASP family verprolin-homologous protein-2 (WAVE2) protein and delivers signals that activate Rap1, regulate both clustering and affinity maturation of integrins, and promote integrin-dependent cell adhesion (15). In addition, overexpressed CrkL was found to increase cell adhesion to fibronectin, a process that requires a functional CrkL-SH3N domain $(54,55)$.

The CasL docking protein (56) is linked to the $\beta 1$-integrin signaling which provides a costimulus for TCR/CD3-driven T-cell proliferation (57). T cells expressing low levels of CasL exhibited impaired $\beta 1$-integrin-mediated costimulation, a defect that was restored by cell transfection of CasL (58). In TCR-activated T cells, CasL undergoes tyrosine phosphorylation at multiple Y-D$\mathrm{x}-\mathrm{P}$ motifs, which serve high-affinity docking sites for Crk-SH2 domains $(8,47,58)$. The CasL-Crk complex, in turn, recruits the guanine nucleotide exchange factor (GEF), C3G, which effectively catalyzes the guanine nucleotide exchange reaction for Rap1 (21).

The important role of Crk in coordinating C3G-CasL-Rap1 signals leading to integrin-dependent $\mathrm{T}$-cell adhesion and migration was further substantiated by analysis of T cells from Crk and CrkL gene conditional knockout mice (21). These T cells were impaired in their ability to activate Rap1 and responded to chemokines by increased adhesion and migration to sites of inflammation.

While studies on lymphocytes emphasized the role of Crk in the regulation of the $\mathrm{C} 3 \mathrm{G}$-dependent activation of Rap1, other studies demonstrated that Crk regulates additional Rho family GTPases (59-61) and involved in the regulation of cell migration by coordinating stimulus-dependent reorganization of the actin cytoskeleton $(61,62)$.

Recent studies demonstrated that the Abl kinases, as well as the CasL and Hef1-associated signal transducer in hematopoietic cells (Chat-H), are integral components of the signaling pathway that promotes Rap1 activation and chemokine-induced Tcell migration $(63,64)$. Abl kinases, Abl and Arg, are known to be activated in response to TCR engagement and contribute to maximal TCR signaling (65). In addition, they are activated downstream of the SDF- $1 \alpha$ chemokine receptor and control the tyrosine phosphorylation of CasL, an upstream regulator of Rap1 
(63). Thus, by coupling the regulation of CasL and Rapl effector proteins to chemokine receptors, the Abl/Arg kinases link chemokine signals to T-cell polarization and migration. In agreement with the above findings, loss of Abl and Arg in mouse T cells inhibited their homeostatic migration and ability to recruit to sites of inflammation (63).

The Chat- $\mathrm{H}$ is a member of the novel $\mathrm{SH} 2$-containing protein (NSP) family (66), also known as the hematopoietic organ-specific NSP3 isoform. It operates upstream of Rap1 and is required for both basal and chemokine-induced phosphorylation of CasL. Chat-H-depleted T cells exhibited diminished chemokineinduced activation of Rap1 that coincided with impaired integrinmediated adhesion and migration. It is therefore assumed that the effector function of Chat-H involves the coupling of the chemokine receptor signaling pathways to integrin-mediated Tcell adhesion (64).

The ZAP-70 PTK is a Crk-binding protein that critically involved in TCR signaling and also regulates integrin receptor function (67-71). TCR stimulation of ZAP-70-negative T cells failed to induce $\beta 1$-integrin-dependent T-cell adhesion to fibronectin, a defect that was corrected by the reintroduction of ZAP-70 to the cells (67). In addition, TCR activation-dependent binding of the lymphocyte function-associated antigen 1 (LFA-1) integrin to its ligand, intercellular adhesion molecule 1 (ICAM1), initiates the delivery of "inside-out" signals to the LFA-1 in a ZAP-70-dependent manner (69). In these studies, overexpression of a dominant-negative ZAP-70 in a T-cell hybridoma blocked the LFA-1-dependent in vitro invasion of the hybridoma cells, inhibited their ability to disseminate in vivo and form remote metastases, and suppressed their ability to migrate toward the chemokine, stromal cell-derived factor 1 (SDF-1) (69). ZAP-70 associates both with the TCR and LFA-1 receptors $(68,70,72)$ and is essential for the delivery of "outside-in" and "inside-out" signals leading, among other things, to T-cell adhesion. Since Crk proteins interact with tyrosine phosphorylated and activated ZAP-70 in a T-cell activation-dependent manner $(11,12,73)$, the physical interaction of ZAP-70 with Crk might be required for the coupling of ZAP-70 to effector molecules operating downstream of activated TCR and/or integrin receptors. In such a case, TCR activation-dependent Crk binding to ZAP-70 might contribute both to "outside-in" and "inside-out" signals that upregulate integrin-mediated T-cell adhesion. This model is partially supported by findings from two independent studies, showing that ZAP-70 with a Phe-to-Tyr substitution at position 315 abolishes both CrkII binding to ZAP-70 (11) and TCR activationdependent increase in $\beta 1$-integrin-mediated cell adhesion (71). Further studies with Crk-SH2 mutants deficient in their ability to interact with ZAP-70 are needed in order to resolve this issue.

As described earlier, one of the modes of regulation of CrkII conformation and function involves its cis-trans isomerization controlled by immunophilins $(28,29,37)$. Our studies revealed that a combination of immunophilin inhibitory drugs, which includes CsA and FK506, interferes with C3G binding to CrkII (14). This, in turn, impaired the integrin-associated signaling and inhibited T-cell adhesion to fibronectin as well as migration toward the chemokine, SDF-1 $\alpha$ (14). We found that overexpression of either CrkI or CrkII increased T-cell adhesion and invasion. However, inhibitors of immunophilin suppressed the ability of CrkII-, but not CrkI-overexpressing Jurkat $\mathrm{T}$ cells to adhere to fibronectin-coated surfaces and migrate toward SDF-1 $\alpha(14)$.

An additional effector molecule that regulates T-cell adhesion by alteration of Crk-dependent signaling pathways is the E3ligase, $\mathrm{Cbl}$ (74). The Cbl protein catalyzes protein ubiquitination and is involved in the regulation of TCR-coupled signals that induce T-cell adhesion. Upon activation of T cells, Cbl undergoes tyrosine phosphorylation and associates with the Crk-SH2 domain and $\mathrm{p} 85$, the regulatory subunit of the phosphoinositide 3-kinase (PI3-K), forming a trimolecular protein complex (74). Cbl-b binding to CrkL induces the ubiquitination of CrkL, while knockdown of Cbl-b results in an increase in several parameters relevant to T-cell adhesion, including the association of CrkL and C3G, activity of the small GTPase, Rap1, and clustering of LFA-1, in response to TCR triggering $(75,76)$. The results suggest that Cbl-b is a negative regulator of the CrkL-C3G-mediated Rap1 and LFA-1 activation of $\mathrm{T}$ cells and of Crk-regulated signaling events that promote T-cell adhesion $(75,76)$. Protein complexes consisting of $\mathrm{Crk}$ and $\mathrm{Cbl}$ are also involved in signaling from the L-selectin (CD62) adhesion receptors (77) as well as the CXCR4 chemokine receptor (78). Taken together, the results suggest that Cbl-mediated regulation of Crk-dependent signals is an important element in the regulation of T-lymphocyte adhesion, migration, and homing (79).

\section{Concluding Remarks and Future Directions}

The Crk adaptor proteins play an essential role in the regulation of $\mathrm{T}$-cell adhesion and migration to sites of inflammation. Together with C3G and CasL, they coordinate signaling pathways leading to activation of Rap1 that upregulate the clustering and affinity of integrins to their ligands. This general mechanism might be relevant to the regulation of some, but not all, integrins since different T-cell integrins might be coupled to distinct effector proteins. The mechanism of regulation of the stoichiometric ratio between CrkI, CrkII, and CrkL in specific cell types is unknown, and the contribution of individual Crk isoforms to the adhesion response has not been thoroughly studied. Further investigations are also required in order to determine the potential regulation of selected Crk proteins by isomerases and whether inhibitors of immunophilins can downregulate T-cell adhesion and migration irrespective of their inhibition of the calcineurin/NFAT pathway.

\section{Acknowledgments}

Work in our laboratory was supported by the USA-Israel Binational Science Foundation, the Israel Science Foundation administered by the Israel Academy of Science and Humanities, and a donation by Mr. Martin Kolinsky. NI holds the Joseph H. Krupp Chair in Cancer Immunobiology. 


\section{References}

1. Mayer BJ, Hamaguchi M, Hanafusa H. A novel viral oncogene with structural similarity to phospholipase C. Nature (1988) 332:272-5. doi:10.1038/332272a0

2. Birge RB, Kalodimos C, Inagaki F, Tanaka S. Crk and CrkL adaptor proteins: networks for physiological and pathological signaling. Cell Commun Signal (2009) 7:13. doi:10.1186/1478-811X-7-13

3. Gelkop S, Babichev Y, Kalifa R, Tamir A, Isakov N. Involvement of crk adapter proteins in regulation of lymphoid cell functions. Immunol Res (2003) 28:79-91. doi:10.1385/IR:28:2:79

4. Feller SM. Crk family adaptors-signalling complex formation and biological roles. Oncogene (2001) 20:6348-71. doi:10.1038/sj.onc.1204779

5. Kumar S, Fajardo JE, Birge RB, Sriram G. Crk at the quarter century mark: perspectives in signaling and cancer. J Cell Biochem (2014) 115:819-25. doi:10. $1002 /$ jcb. 24749

6. Liu D. The adaptor protein Crk in immune response. Immunol Cell Biol (2014) 92:80-9. doi:10.1038/icb.2013.64

7. Songyang Z, Shoelson SE, Chaudhuri M, Gish G, Pawson T, Haser WG, et al. SH2 domains recognize specific phosphopeptide sequences. Cell (1993) 72:767-78. doi:10.1016/0092-8674(93)90404-E

8. Kanda H, Mimura T, Morino N, Hamasaki K, Nakamoto T, Hirai H, et al. Ligation of the $\mathrm{T}$ cell antigen receptor induces tyrosine phosphorylation of p105CasL, a member of the p130Cas-related docking protein family, and its subsequent binding to the Src homology 2 domain of c-Crk. Eur J Immunol (1997) 27:2113-7. doi:10.1002/eji.1830270840

9. Sawasdikosol S, Ravichandran KS, Lee KK, Chang JH, Burakoff SJ. Crk interacts with tyrosine-phosphorylated p116 upon T cell activation. J Biol Chem (1995) 270:2893-6. doi:10.1074/jbc.270.7.2893

10. Sawasdikosol S, Chang JH, Pratt JC, Wolf G, Shoelson SE, Burakoff SJ. Tyrosinephosphorylated Cbl binds to Crk after T cell activation. J Immunol (1996) 157:110-6.

11. Gelkop S, Gish GD, Babichev Y, Pawson T, Isakov N. T cell activationinduced CrkII binding to the Zap70 protein tyrosine kinase is mediated by Lck-dependent phosphorylation of Zap70 tyrosine 315. J Immunol (2005) 175:8123-32. doi:10.4049/jimmunol.175.12.8123

12. Gelkop S, Isakov N. T cell activation stimulates the association of enzymatically active tyrosine-phosphorylated ZAP-70 with the Crk adapter proteins. J Biol Chem (1999) 274:21519-27. doi:10.1074/jbc.274.31.21519

13. Isakov $\mathrm{N}$. Role of immunoreceptor tyrosine-based activation motif in signal transduction from antigen and $\mathrm{Fc}_{\mathrm{C}}$ receptors. Adv Immunol (1998) 69:183-247. doi:10.1016/S0065-2776(08)60608-2

14. Nath PR, Dong G, Braiman A, Isakov N. Immunophilins control T lymphocyte adhesion and migration by regulating CrkII binding to C3G. J Immunol (2014) 193:3966-77. doi:10.4049/jimmunol.1303485

15. Nolz JC, Nacusi LP, Segovis CM, Medeiros RB, Mitchell JS, Shimizu Y, et al. The WAVE2 complex regulates T cell receptor signaling to integrins via Abland CrkL-C3G-mediated activation of Rap1. J Cell Biol (2008) 182:1231-44. doi:10.1083/jcb.200801121

16. Brownlie RJ, Zamoyska R. T cell receptor signalling networks: branched, diversified and bounded. Nat Rev Immunol (2013) 13:257-69. doi:10.1038/nri3403

17. Wu X, Knudsen B, Feller SM, Zheng J, Sali A, Cowburn D, et al. Structural basis for the specific interaction of lysine-containing proline-rich peptides with the N-terminal SH3 domain of c-Crk. Structure (1995) 3:215-26. doi:10.1016/ S0969-2126(01)00151-4

18. Reedquist KA, Fukazawa T, Panchamoorthy G, Langdon WY, Shoelson SE, Druker BJ, et al. Stimulation through the $\mathrm{T}$ cell receptor induces $\mathrm{Cbl}$ association with Crk proteins and the guanine nucleotide exchange protein C3G. J Biol Chem (1996) 271:8435-42. doi:10.1074/jbc.271.14.8435

19. Oehrl W, Kardinal C, Ruf S, Adermann K, Groffen J, Feng GS, et al. The germinal center kinase (GCK)-related protein kinases HPK1 and KHS are candidates for highly selective signal transducers of Crk family adapter proteins. Oncogene (1998) 17:1893-901. doi:10.1038/sj.onc.1202108

20. Ling P, Yao Z, Meyer CF, Wang XS, Oehrl W, Feller SM, et al. Interaction of hematopoietic progenitor kinase 1 with adapter proteins Crk and CrkL leads to synergistic activation of c-Jun N-terminal kinase. Mol Cell Biol (1999) 19:1359-68.

21. Huang Y, Clarke F, Karimi M, Roy NH, Williamson EK, Okumura M, et al. CRK proteins selectively regulate $\mathrm{T}$ cell migration into inflamed tissues. J Clin Invest (2015) 125:1019-32. doi:10.1172/JCI77278
22. Park TJ, Curran T. Crk and Crk-like play essential overlapping roles downstream of disabled-1 in the Reelin pathway. J Neurosci (2008) 28:13551-62. doi:10.1523/JNEUROSCI.4323-08.2008

23. Muralidharan V, Dutta K, Cho J, Vila-Perello M, Raleigh DP, Cowburn D, et al. Solution structure and folding characteristics of the $\mathrm{C}$-terminal SH3 domain of c-Crk-II. Biochemistry (2006) 45:8874-84. doi:10.1021/bi060590z

24. Smith JJ, Richardson DA, Kopf J, Yoshida M, Hollingsworth RE, Kornbluth S. Apoptotic regulation by the Crk adapter protein mediated by interactions with Wee1 and Crm1/exportin. Mol Cell Biol (2002) 22:1412-23. doi:10.1128/MCB. 22.5.1412-1423.2002

25. Harkiolaki M, Gilbert RJ, Jones EY, Feller SM. The C-terminal SH3 domain of CRKL as a dynamic dimerization module transiently exposing a nuclear export signal. Structure (2006) 14:1741-53. doi:10.1016/j.str.2006.09.013

26. Ogawa S, Toyoshima H, Kozutsumi H, Hagiwara K, Sakai R, Tanaka T, et al. The C-terminal SH3 domain of the mouse c-Crk protein negatively regulates tyrosine-phosphorylation of Crk associated p130 in rat 3Y1 cells. Oncogene (1994) 9:1669-78.

27. Kobashigawa Y, Sakai M, Naito M, Yokochi M, Kumeta H, Makino Y, et al. Structural basis for the transforming activity of human cancer-related signaling adaptor protein CRK. Nat Struct Mol Biol (2007) 14:503-10. doi:10.1038/ nsmb1241

28. Sarkar P, Reichman C, Saleh T, Birge RB, Kalodimos CG. Proline cis-trans isomerization controls autoinhibition of a signaling protein. Mol Cell (2007) 25:413-26. doi:10.1016/j.molcel.2007.01.004

29. Sarkar P, Saleh T, Tzeng SR, Birge RB, Kalodimos CG. Structural basis for regulation of the Crk signaling protein by a proline switch. Nat Chem Biol (2011) 7:51-7. doi:10.1038/nchembio.494

30. de Jong R, ten Hoeve J, Heisterkamp N, Groffen J. Tyrosine 207 in CRKL is the BCR/ABL phosphorylation site. Oncogene (1997) 14:507-13. doi:10.1038/ sj.onc. 1200885

31. Peterson ME, Long EO. Inhibitory receptor signaling via tyrosine phosphorylation of the adaptor Crk. Immunity (2008) 29:578-88. doi:10.1016/j.immuni. 2008.07.014

32. Feller SM, Knudsen B, Hanafusa H. c-Abl kinase regulates the protein binding activity of c-Crk. $E M B O J$ (1994) 13:2341-51.

33. Seo JH, Wood LJ, Agarwal A, O'Hare T, Elsea CR I, Griswold J, et al. A specific need for CRKL in p210BCR-ABL-induced transformation of mouse hematopoietic progenitors. Cancer Res (2010) 70:7325-35. doi:10.1158/00085472.CAN-10-0607

34. Rosen MK, Yamazaki T, Gish GD, Kay CM, Pawson T, Kay LE. Direct demonstration of an intramolecular SH2-phosphotyrosine interaction in the Crk protein. Nature (1995) 374:477-9. doi:10.1038/374477a0

35. Anafi M, Rosen MK, Gish GD, Kay LE, Pawson T. A potential SH3 domainbinding site in the Crk SH2 domain. J Biol Chem (1996) 271:21365-74. doi:10. $1074 /$ jbc.271.35.21365

36. Donaldson LW, Gish G, Pawson T, Kay LE, Forman-Kay JD. Structure of a regulatory complex involving the $\mathrm{Abl} \mathrm{SH} 3$ domain, the $\mathrm{Crk} \mathrm{SH} 2$ domain, and a Crk-derived phosphopeptide. Proc Natl Acad Sci U S A (2002) 99:14053-8. doi:10.1073/pnas.212518799

37. Isakov N. A new twist to adaptor proteins contributes to regulation of lymphocyte cell signaling. Trends Immunol (2008) 29:388-96. doi:10.1016/j.it.2008.04. 006

38. Schmidpeter PA, Schmid FX. Molecular determinants of a regulatory prolyl isomerization in the signal adapter protein c-CrkII. ACS Chem Biol (2014) 9:1145-52. doi:10.1021/cb500001n

39. Jankowski W, Saleh T, Pai MT, Sriram G, Birge RB, Kalodimos CG. Domain organization differences explain Bcr-Abl's preference for CrkL over CrkII. Nat Chem Biol (2012) 8:590-6. doi:10.1038/nchembio.954

40. Kobashigawa Y, Inagaki F. Structural biology: CrkL is not Crk-like. Nat Chem Biol (2012) 8:504-5. doi:10.1038/nchembio.963

41. Gong XH, Chen C, Hou P, Zhu SC, Wu CQ, Song CL, et al. Overexpression of miR-126 inhibits the activation and migration of HSCs through targeting CRK. Cell Physiol Biochem (2014) 33:97-106. doi:10.1159/000356653

42. Harris ES, Weyrich AS, Zimmerman GA. Lessons from rare maladies: leukocyte adhesion deficiency syndromes. Curr Opin Hematol (2013) 20:16-25. doi:10. 1097/MOH.0b013e32835a0091

43. Springer TA, Dustin ML. Integrin inside-out signaling and the immunological synapse. Curr Opin Cell Biol (2012) 24:107-15. doi:10.1016/j.ceb.2011.10.004 
44. Evans R, Patzak I, Svensson L, De Filippo K, Jones K, McDowall A, et al. Integrins in immunity. J Cell Sci (2009) 122:215-25. doi:10.1242/jcs.019117

45. Petruzzelli L, Takami M, Herrera R. Adhesion through the interaction of lymphocyte function-associated antigen-1 with intracellular adhesion molecule-1 induces tyrosine phosphorylation of p130cas and its association with c-CrkII. J Biol Chem (1996) 271:7796-801. doi:10.1074/jbc.271.13.7796

46. Kloog Y, Mor A. Cytotoxic-T-lymphocyte antigen 4 receptor signaling for lymphocyte adhesion is mediated by C3G and Rap1. Mol Cell Biol (2014) 34:978-88. doi:10.1128/MCB.01024-13

47. Ohashi Y, Tachibana K, Kamiguchi K, Fujita H, Morimoto C. T cell receptormediated tyrosine phosphorylation of Cas- $\mathrm{L}$, a $105-\mathrm{kDa}$ Crk-associated substrate-related protein, and its association of Crk and C3G. J Biol Chem (1998) 273:6446-51. doi:10.1074/jbc.273.11.6446

48. Knudsen BS, Feller SM, Hanafusa H. Four proline-rich sequences of the guanine-nucleotide exchange factor $\mathrm{C} 3 \mathrm{G}$ bind with unique specificity to the first Src homology 3 domain of Crk. J Biol Chem (1994) 269:32781-7.

49. Tanaka S, Morishita T, Hashimoto Y, Hattori S, Nakamura S, Shibuya M, et al. C3G, a guanine nucleotide-releasing protein expressed ubiquitously, binds to the Src homology 3 domains of CRK and GRB2/ASH proteins. Proc Natl Acad Sci U S A (1994) 91:3443-7. doi:10.1073/pnas.91.8.3443

50. Gotoh T, Hattori S, Nakamura S, Kitayama H, Noda M, Takai Y, et al. Identification of Rap1 as a target for the $\mathrm{Crk} \mathrm{SH} 3$ domain-binding guanine nucleotidereleasing factor C3G. Mol Cell Biol (1995) 15:6746-53.

51. Bos JL. Linking rap to cell adhesion. Curr Opin Cell Biol (2005) 17:123-8. doi:10.1016/j.ceb.2005.02.009

52. Tanaka S, Ouchi T, Hanafusa H. Downstream of Crk adaptor signaling pathway: activation of Jun kinase by v-Crk through the guanine nucleotide exchange protein C3G. Proc Natl Acad Sci U S A (1997) 94:2356-61. doi:10.1073/pnas. 94.6.2356

53. Katagiri K, Hattori M, Minato N, Irie S, Takatsu K, Kinashi T. Rap1 is a potent activation signal for leukocyte function-associated antigen 1 distinct from protein kinase $\mathrm{C}$ and phosphatidylinositol-3-OH kinase. Mol Cell Biol (2000) 20:1956-69. doi:10.1128/MCB.20.6.1956-1969.2000

54. Uemura N, Griffin JD. The adapter protein Crkl links Cbl to C3G after integrin ligation and enhances cell migration. J Biol Chem (1999) 274:37525-32. doi:10. 1074/jbc.274.53.37525

55. Arai A, Nosaka Y, Kohsaka H, Miyasaka N, Miura O. CrkL activates integrinmediated hematopoietic cell adhesion through the guanine nucleotide exchange factor C3G. Blood (1999) 93:3713-22.

56. Seo S, Ichikawa M, Kurokawa M. Structure and function of cas-L and integrin-mediated signaling. Crit Rev Immunol (2006) 26:391-406. doi:10.1615/ CritRevImmunol.v26.i5.20

57. Hunter AJ, Shimizu Y. Alpha 4 beta 1 integrin-mediated tyrosine phosphorylation in human T cells: characterization of Crk- and Fyn-associated substrates (pp105, pp115, and human enhancer of filamentation-1) and integrindependent activation of p59fyn1. J Immunol (1997) 159:4806-14.

58. Iwata S, Ohashi Y, Kamiguchi K, Morimoto C. Beta 1-integrin-mediated cell signaling in T lymphocytes. J Dermatol Sci (2000) 23:75-86. doi:10.1016/S09231811(99)00096-1

59. Nakamura T, Komiya M, Sone K, Hirose E, Gotoh N, Morii H, et al. Grit, a GTPase-activating protein for the Rho family, regulates neurite extension through association with the TrkA receptor and N-Shc and CrkL/Crk adapter molecules. Mol Cell Biol (2002) 22:8721-34. doi:10.1128/MCB.22.24.87218734.2002

60. Altun-Gultekin Z, Chandriani FS, Bougeret C, Ishizaki T, Narumiya S, de Graaf $P$, et al. Activation of Rho-dependent cell spreading and focal adhesion biogenesis by the v-Crk adaptor protein. Mol Cell Biol (1998) 18:3044-58.

61. Antoku S, Mayer BJ. Distinct roles for Crk adaptor isoforms in actin reorganization induced by extracellular signals. J Cell Sci (2009) 122:4228-38. doi:10. $1242 /$ jcs.054627

62. Bougneres L, Girardin SE, Weed SA, Karginov AV, Olivo-Marin JC, Parsons JT, et al. Cortactin and Crk cooperate to trigger actin polymerization during Shigella invasion of epithelial cells. J Cell Biol (2004) 166:225-35. doi:10.1083/ jcb. 200402073

63. Gu JJ, Lavau CP, Pugacheva E, Soderblom EJ, Moseley MA, Pendergast AM. Abl family kinases modulate $\mathrm{T}$ cell-mediated inflammation and chemokine-induced migration through the adaptor HEF1 and the GTPase Rap1. Sci Signal (2012) 5:ra51. doi:10.1126/scisignal.2002632

64. Regelmann AG, Danzl NM, Wanjalla C, Alexandropoulos K. The hematopoietic isoform of Cas-Hefl-associated signal transducer regulates chemokine-induced inside-out signaling and T cell trafficking. Immunity (2006) 25:907-18. doi:10. 1016/j.immuni.2006.09.014

65. Gu JJ, Ryu JR, Pendergast AM. Abl tyrosine kinases in T-cell signaling. Immunol Rev (2009) 228:170-83. doi:10.1111/j.1600-065X.2008.00751.x

66. Wallez Y, Mace PD, Pasquale EB, Riedl SJ. NSP-CAS protein complexes: emerging signaling modules in cancer. Genes Cancer (2012) 3:382-93. doi:10.1177/ 1947601912460050

67. Epler JA, Liu R, Chung H, Ottoson NC, Shimizu Y. Regulation of beta 1 integrinmediated adhesion by $\mathrm{T}$ cell receptor signaling involves ZAP-70 but differs from signaling events that regulate transcriptional activity. J Immunol (2000) 165:4941-9. doi:10.4049/jimmunol.165.9.4941

68. Evans R, Lellouch AC, Svensson L, McDowall A, Hogg N. The integrin LFA1 signals through ZAP-70 to regulate expression of high-affinity LFA-1 on T lymphocytes. Blood (2011) 117:3331-42. doi:10.1182/blood-2010-06-289140

69. Soede RD, Wijnands YM, Van Kouteren-Cobzaru I, Roos E. ZAP-70 tyrosine kinase is required for LFA-1-dependent T cell migration. J Cell Biol (1998) 142:1371-9. doi:10.1083/jcb.142.5.1371

70. Soede RD, Driessens MH, Ruuls-Van Stalle L, Van Hulten PE, Brink A, Roos E. LFA-1 to LFA-1 signals involve zeta-associated protein-70 (ZAP-70) tyrosine kinase: relevance for invasion and migration of a T cell hybridoma. J Immunol (1999) 163:4253-61.

71. Goda S, Quale AC, Woods ML, Felthauser A, Shimizu Y. Control of TCRmediated activation of beta 1 integrins by the ZAP-70 tyrosine kinase interdomain B region and the linker for activation of T cells adapter protein. J Immunol (2004) 172:5379-87. doi:10.4049/jimmunol.172.9.5379

72. Chan AC, Iwashima M, Turck CW, Weiss A. ZAP-70: a $70 \mathrm{kd}$ protein-tyrosine kinase that associates with the TCR zeta chain. Cell (1992) 71:649-62. doi:10. 1016/0092-8674(92)90598-7

73. Sasahara Y, Rachid R, Byrne MJ, de la Fuente MA, Abraham RT, Ramesh N, et al. Mechanism of recruitment of WASP to the immunological synapse and of its activation following TCR ligation. Mol Cell (2002) 10:1269-81. doi:10.1016/ S1097-2765(02)00728-1

74. Gelkop S, Babichev Y, Isakov N. T cell activation induces direct binding of the Crk adapter protein to the regulatory subunit of phosphatidylinositol 3-kinase (p85) via a complex mechanism involving the Cbl protein. J Biol Chem (2001) 276:36174-82. doi:10.1074/jbc.M100731200

75. Shao Y, Elly C, Liu YC. Negative regulation of Rap1 activation by the Cbl E3 ubiquitin ligase. EMBO Rep (2003) 4:425-31. doi:10.1038/sj.embor.embor813

76. Zhang W, Shao Y, Fang D, Huang J, Jeon MS, Liu YC. Negative regulation of T cell antigen receptor-mediated Crk-L-C3G signaling and cell adhesion by Cbl-b. J Biol Chem (2003) 278:23978-83. doi:10.1074/jbc.M212671200

77. Brenner B, Kadel S, Birle A, Linderkamp O. L-selectin tyrosine phosphorylates $\mathrm{cbl}$ and induces association of tyrosine-phosphorylated cbl with crkl and grb2. Biochem Biophys Res Commun (2001) 282:41-7. doi:10.1006/bbrc.2001.4546

78. Chernock RD, Cherla RP, Ganju RK. SHP2 and cbl participate in alphachemokine receptor CXCR4-mediated signaling pathways. Blood (2001) 97:608-15. doi:10.1182/blood.V97.3.608

79. Uemura N, Salgia R, Ewaniuk DS, Little MT, Griffin JD. Involvement of the adapter protein CRKL in integrin-mediated adhesion. Oncogene (1999) 18:3343-53. doi:10.1038/sj.onc.1202689

Conflict of Interest Statement: The authors declare that the research was conducted in the absence of any commercial or financial relationships that could be construed as a potential conflict of interest.

Copyright (C) 2015 Braiman and Isakov. This is an open-access article distributed under the terms of the Creative Commons Attribution License (CC BY). The use, distribution or reproduction in other forums is permitted, provided the original author(s) or licensor are credited and that the original publication in this journal is cited, in accordance with accepted academic practice. No use, distribution or reproduction is permitted which does not comply with these terms. 